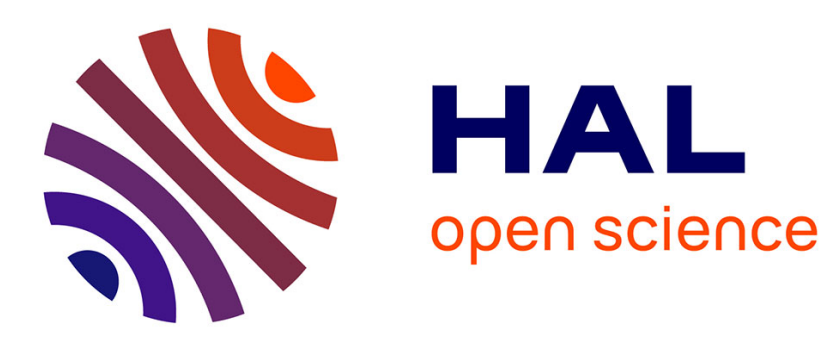

\title{
Solving the Langevin equation with stochastic algebraically correlated noise
}

\author{
M. Ploszajczak, T. Srokowski
}

\section{To cite this version:}

M. Ploszajczak, T. Srokowski. Solving the Langevin equation with stochastic algebraically correlated noise. Physical Review E: Statistical, Nonlinear, and Soft Matter Physics, 1997, 55, pp.5126-5133. 10.1103/PhysRevE.55.5126 . in2p3-00021696

\section{HAL Id: in2p3-00021696 https://hal.in2p3.fr/in2p3-00021696}

Submitted on 14 Apr 2014

HAL is a multi-disciplinary open access archive for the deposit and dissemination of scientific research documents, whether they are published or not. The documents may come from teaching and research institutions in France or abroad, or from public or private research centers.
L'archive ouverte pluridisciplinaire HAL, est destinée au dépôt et à la diffusion de documents scientifiques de niveau recherche, publiés ou non, émanant des établissements d'enseignement et de recherche français ou étrangers, des laboratoires publics ou privés. 

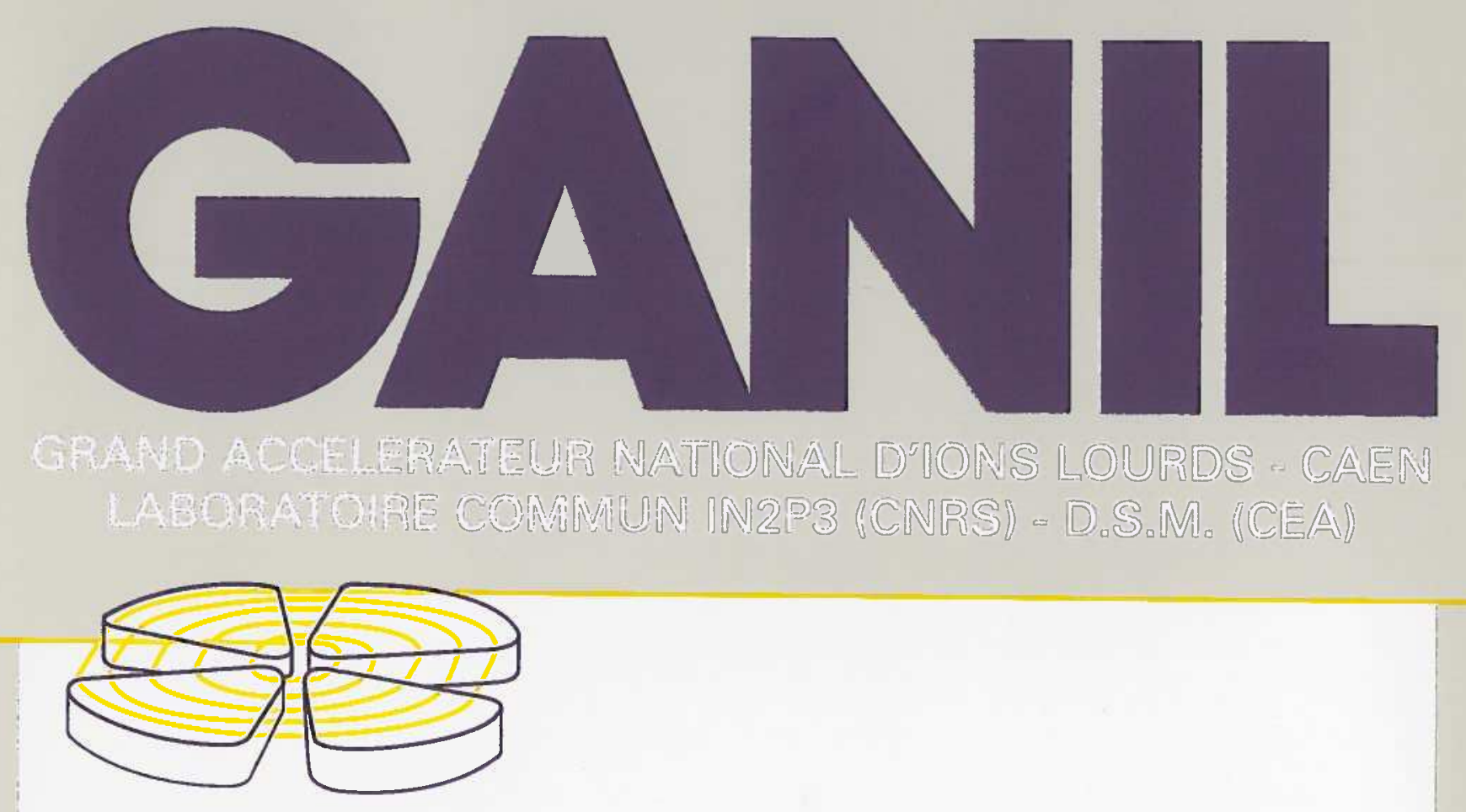

Solving Langevin Equation with the Stochastic Algebraically Correlated Noise

\author{
M. Płoszajczak ${ }^{\dagger}$ and T. Srokowski ${ }^{\ddagger}$ \\ † Grand Accélérateur National d'Ions Lourds (GANIL), \\ CEA/DSM - CNRS/IN2P3, BP 5027, F-14021 Caen Cedex, France \\ and \\ $\ddagger$ Institute of Nuclear Physics, PL - 31-342 Kraków, Poland
}




\title{
Solving Langevin Equation with the Stochastic Algebraically Correlated Noise
}

\author{
M. Ptoszajczak ${ }^{\dagger}$ and T. Srokowski ${ }^{\ddagger}$ \\ † Grand Accélérateur National d'Ions Lourds (GANIL), \\ CEA/DSM - CNRS/IN2P3, BP 5027, F-14021 Caen Cedex, France \\ and \\ ‡ Institute of Nuclear Physics, PL - 31-342 Kraków, Poland
} (May 17, 1996)

\begin{abstract}
Long time tail in the velocity and force autocorrelation function has been found recently in the molecular dynamics simulations of the peripheral collisions of ions. Simulation of those slowly decaying correlations in the stochastic transport theory, requires the development of new methods of generating stochastic force of arbitrarily long correlation times. In this paper we propose the Markovian process, the multidimensional Kangaroo process, which permits describing various algebraic correlated stochastic processes.

PACS numbers: $05.40 .+\mathrm{j}, 05.45 .+\mathrm{b}, 05.60+\mathrm{w}$
\end{abstract}

Typeset using REVT $\mathrm{E} X$ 


\section{INTRODUCTION}

Dynamics of a classical many body system can be investigated using either the molecular dynamics approach or the kinetic rate equations. Both approaches can be generalized to incorporate also the Pauli exclusion principle for fermions. In the latter case, one considers for example different variants of the Boltzmann or Boltzmann-Langevin equations, whereas in the former case the 'quantal' version of the molecular dynamics, the so called antisymmetrized molecular dynamics [1] has been proposed. Chaotic properties of atomic nuclei have been discussed in the framework of the classical molecular dynamics (CMD). For central collisions where fusion process dominates, it has been demonstrated $[2,3]$ that both the velocity autocorrelation function : $C(t)=\left\langle\mathbf{v}\left(t_{0}\right) \mathbf{v}\left(t_{0}+t\right)>\right.$ and the force autocorrelation function : $\tilde{C}(t)=<\mathbf{F}\left(t_{0}\right) \mathbf{F}(t)>$, decay exponentially in time. The equilibration time is short, allowing the statistical properties of the compound nucleus to show up at the early stage of the reaction. On the contrary, in the peripheral collisions of ions, the algebraic, long time tail $\sim t^{-\gamma}(\gamma=1)$ was found in both the velocity and force autocorrelation functions $[2,3]$. Moreover, the survival probability is given by the powerlaw [4] .

The Fourier transform of $C(t)$ gives the power spectrum $S(\omega)$. For the peripheral collisions [2],$C(t) \sim t^{-1}$ and, hence, $S(\omega) \sim|\ln \omega|$. The mean square displacement in configurational space is in this case [2]: $\sigma^{2}(t) \equiv\left\langle\left(\mathbf{r}(t)-\mathbf{r}\left(t_{0}\right)\right)^{2}\right\rangle \propto t \ln \frac{t}{t_{0}}-t+t_{0}$. The diffusion is anomalously enhanced (superdiffusion) and the diffusion rate $: D \equiv \lim _{t \rightarrow \infty} \sigma^{2}(t) / t \mathrm{di}$ verges logarithmically, i.e. the dissipation rate does not stabilize, as it would be the case for a normal diffusion ( $D=$ const). The same dependence holds also for the mean-square displacement in the velocity space.

The logarithmic power spectrum and the enhanced diffusion has been found for the periodic Lorentz gas (PLG) of hard discs (the extended Sinai billiard) [5]. From the point of view of transport phenomena, many physical systems can be reduced to a simple lattice of periodic potentials. Besides the CMD in the orbiting regime, the dynamics of electrons in crystals moving in a magnetic field or the ballistic-electron dynamics in lateral superlattices 
, are other examples which can be modelled in terms of periodic $2 D$ lattices $[6,7]$. The similarity of the diffusive behaviour for systems as different as the CMD and the PLG, follows from the fact that the powerlaw tail of the velocity autocorrelation function is due to the existence of long free paths. This behaviour is universal and insensitive to the details of the potential, in particular to its short distance features. Such universality allows to describe phenomena involving long free paths in a framework of the Langevin equation with algebraically correlated noise [3]. Inclusion of effects connected with the antisymmetrization of the wave function for fermions does not modify this picture qualitatively. The nonlocality of the Pauli potential destroys cantori in the phase space and the diffusion process, for sufficiently large lattice spacing, is dominated by long free paths and hence its power spectrum is logarithmic at small frequency limit [8]. This finding makes the purely classical description more reliable.

The relevance of the Langevin approach for the description of an induced fission process has been realized long time ago [9]. The slow collective motion with its high mass parameter is treated as a Brownian particle, whereas the fast nucleonic degrees of freedom form the heat bath. In generalized Brownian motion theory [10], the Hamilton equations can be rewritten in the form of the Langevin equation by making use of the projection operator technique. The total force acting on a Brownian particle is divided into a systematic part and a random part. The slowly varying part describes the evolution of macroscopic variables. The fast varying part leads to the fluctuations around the most probable path. In the conventional Langevin approach, it is usually assumed that the time-evolution of the fast varying random part is stochastic and the time rate of changes is much faster than that of the systematic part. Consequently, it is assumed that the correlation function of the random part is $\delta$ - correlated or decays exponentially. (For a recent review of stochastic theories with the colored noise see ref [11] .)

A hypothesis of the rapid decay of the force correlations holds for central collisions and the CMD yields in this case fast decaying correlations in both velocity and acceleration (force) [2] . However, for peripheral reactions and/or strongly elongated shapes, the correlations 
decay algebraically [2] . Such slowly decaying correlations are known in various phenomena including the chemical reactions in solutions [12], ligands migration in biomolecules [13] , atomic diffusion through a periodic lattice [14], Stark broadening [15] and many others. This regime is certainly beyond the standard Langevin approach and requires the consideration of the colored noise of arbitrarily long correlation time. The first important step in this direction was the theory of line shapes and relaxation in magnetic resonance systems through the study of the so called Kubo oscillator [16] . More recently, the Kubo-Anderson process [17] with the slowly decaying noise correlation function, the so called 'Kangaroo process' (KP) [18], was used to explain the noise-induced Stark broadening [15] .

Recently, we have proposed a new method which extends the Langevin approach for phenomena with either exponentially or algebraically decaying force correlations [2]. In these studies, we have investigated a two-dimensional Langevin equation :

$$
\begin{aligned}
\frac{d \mathbf{r}}{d t} & =\mathbf{v} \\
m \frac{d \mathbf{v}}{d t}(t) & =-\beta \mathbf{v}(t)-\frac{\partial V(r)}{\partial \mathbf{r}}+\mathbf{F}(t)
\end{aligned}
$$

where the potential $V$ generates a conservative force, $\beta$ is the friction constant, and the external noise (stochastic force) $\mathbf{F}(t)$ has algebraically decaying correlations:

$$
\begin{aligned}
<\mathbf{F}(0) \mathbf{F}(t) & >\sim 1 / t \\
<\mathbf{F}(t) & >=0
\end{aligned}
$$

These conditions do not determine the noise uniquely. In our earlier studies, we have proposed to simulate $\mathbf{F}(t)$ by deterministic time series of the particle velocity in the PLG. In the present work, we investigate possibility to simulate algebraic correlated noise $F(t)$ by a Markov process, and for this purpose we shall study the generalized KP. In spite of fundamental differences, there are some similarities between these two realizations of the algebraic noise. For both processess, algebraic correlations involved are due to the existence of 'long free paths', i.e. value of the stochastic process ( velocity of the particle in the case of the PLG) remains constant for long time intervals. 
The main goal of this work is to investigate and compare the Langevin processes for these two different ways of generating of the stochastic force. For that purpose we shall compare the most relevant physical quantities like the energy spectrum of the particles and their escape time distribution obtained with different generators of the external noise $\mathbf{F}(t)$. In chapter II we shall remind the most essential features of the PLG process which can be used to generate both algebraic and exponential correlated deterministic noise [3] . Chapter III is devoted to the discussion of the KP. In sect. III.B we shall discuss the multidimensional , norm-conserving generalization of this stochastic process which can be directly compared with the norm-conserving PLG process. We perform this comparison in chapter IV, solving the Langevin equation (1) for particles escaping from the spherically symmetric potential well. Finally, the most important results of this work are summarized and concluded in chapter V.

\section{PERIODIC LORENTZ GAS AND THE CORRELATIONS FOR OPEN AND CLOSE HORIZONS}

Before discussing the KP and its generalization, we want to remind the most essential properties of the PLG (or the extended Sinai billiard). As said before, the PLG was used to generate event by event the erratic chaotic force acting on Brownian particles [3]. The PLG consists of a single point particle moving in a two-dimensional periodic array of fixed circular scatterers of radius $R$ [19] . The lattice spacing is assumed to be equal to two. The point mass is scattered elastically from scatterers and the particle velocity has a unit length. The particle is reflected upon hitting arc of hard discs or meets the periodic boundary condition when it crosses the straight-line segment between hard discs. The orbit consists of the succession of pairs $\left\{s_{n}\left(s_{0}\right), p_{n}\left(p_{0}\right)\right\}$ corresponding to the $n$th bounce when the initial condition was $\left\{s_{0}, p_{0}\right\}$. This dynamics is a mapping $M$ of the phase space $\{s, p\}$ into itself [20] : 


$$
\left(\begin{array}{c}
s_{n+1} \\
p_{n+1}
\end{array}\right)=M\left(\begin{array}{l}
s_{n} \\
p_{n}
\end{array}\right),
$$

The sequence of iterates (3) is uniquely determined as a function of the initial value. The separation between disks completely determines the behaviour of the system. If $R>1$ ('the high-density regime' of the PLG) then the disks overlap and the particle is trapped in a region bounded by four arcs of circles. This situation corresponds to the closed horizon as the particle trajectory is bound. If $R<1$ ('the low-density regime' of the PLG) the particle sees an infinite horizon and may move to an arbitrarily long distance between subsequent collisions, i.e. the length of free paths is unbounded.

The PLG, both for $R<1$ and $R \geq 1$, belongs to the category of so called K-systems [19] , for which the nearby trajectories diverge exponentially and the metric entropy is positive. This system is known to be ergodic in two dimensions and numerical experiments in higher dimensions also indicate its ergodicity [21] . Despite this, the PLG exhibits longtime correlations which are typically associated with the existence of tori in the phase space. For $R<1$, there exist families of trajectories which do not collide with hard scatterers and correspond to a regular motion. Existence of these families is a reason of long-time tails in the correlation functions: the velocity autocorrelation function changes from a stretched exponential decay [21] for a closed horizon situation to an algebraic decay [22] $\left(C(t) \sim t^{-1}\right)$ for an infinite horizon situation. Consequently, the self-generated diffusion process changes from an ordinary diffusion process $(D(t)=$ const $)$ to a superdiffusive process $(D(t) \sim \log t$ ) when the horizon for a wandering particle is opened. In this latter case, the distribution of free path lengths is algebraic : $N(s) \sim s^{-3}$ for large $s$ [21], independently of the dimensionality of the billiard.

At around $R=1$, many quantities, including the probability density of free path length $N(s)$ and the velocity autocorrelation function $C(t)$, exhibit a critical behaviour which resembles a second-order phase transition. In particular, the correlation length diverges and the length scale disappears. One can also define the order parameter : 


$$
\lim _{t \rightarrow \infty} \frac{<r^{2}(t)>}{t \log t}
$$

which is zero for $R>1$ and changes to a finite value $D_{0}$ for $R<1$.

\section{THE KANGAROO PROCESS}

The step-wise constant random function $m(t)$ is called a Kubo-Anderson process if the jumping times $t_{i}(i=-\infty, \ldots,+\infty)$ are uniformly and independently distributed with density $\nu$ in the interval $(-\infty,+\infty)$, and $m(t)$ is a constant $m(t)=m_{i}$ in the interval $t_{i} \leq t<t_{i+1} \cdot m(t)$ is the stationary Markov process with the probability density $P(m)$. Assuming $<m>=0$, one obtains for the covariance of this process :

$$
\tilde{\Gamma}\left(\left|t-t^{\prime}\right|\right) \equiv<m(t) m\left(t^{\prime}\right)>=<m^{2}>\exp \left(-\nu\left|t-t^{\prime}\right|\right) \text {. }
$$

Both the probability density $P(m)$ and the correlation time $T_{\text {corr }}=\nu^{-1}$ for the KuboAnderson process, may be chosen arbitrarily, however the functional form of the covariance is always exponential.

The study of the problem of stochastic Stark broadening [23], where the covariance is proportional to $1 / t$ and is not integrable, has led to modify the Kubo-Anderson process by requiring that the frequency of jumping times $\nu(m)$ is a function of the value of the process itself. This new process has been called the 'Kangaroo process' (KP). The KP is a step-wise constant Markov process with the stationary transition probability given for infinitesimal time intervals by :

$$
P_{K P}\left(m, \Delta t \mid m^{\prime}, 0\right)=\left\{1-\nu\left(m^{\prime}\right) \Delta t\right\} \delta\left(m-m^{\prime}\right)+Q(m) \nu\left(m^{\prime}\right) \Delta t
$$

where $Q(m)$ is a given probability density to be specified below. $P_{K P} d m$ is the probability that the $\mathrm{KP}$ at time $\Delta t$ is between $m$ and $m+d m$, knowing that it was equal $m^{\prime}$ at time $t=0$. The first term on the rhs of eq. (6) is the probability that no jump occured in the time interval $(0, \Delta t)$. The term $\nu\left(m^{\prime}\right) \Delta t$ is the probability that one jump occured. 
Immediately after such a jump, the probability density of $m$ becomes $Q(m)$. The FockerPlanck equation for the KP reads $[24,18]$ :

$$
\begin{aligned}
\frac{\partial}{\partial t} P(m, t)= & \lim _{\Delta \rightarrow 0}\left\{\int P_{K P}\left(m, \Delta t \mid m^{\prime}, 0\right) P\left(m^{\prime}, t\right) d m^{\prime}-P(m, t)\right\}(\Delta t)^{-1} \\
& \Delta \geq 0 \\
= & -\nu(m) P(m, t)+Q(m) \int \nu\left(m^{\prime}\right) P\left(m^{\prime}, t\right) d m^{\prime} .
\end{aligned}
$$

The stationary probability density $P(m)$ of $m(t)$ is related to $Q(m)$ by :

$$
Q(m)=\frac{\nu(m) P(m)}{\int \nu\left(m^{\prime}\right) P\left(m^{\prime}\right) d m^{\prime}}=\frac{\nu(m) P(m)}{\langle\nu\rangle} .
$$

The calculation of the covariance $\tilde{\Gamma}(t)$ of the KP requires the summation of a series to take into account the possible occurence of an arbitrary number of jumps between 0 and $t$. For that let us calculate the Laplace transform of $\tilde{\Gamma}(t)$ :

$$
\tilde{\Gamma}(z)=\int_{0}^{\infty} \exp (i z t) \tilde{\Gamma}(t) d t
$$

which becomes :

$$
\tilde{\Gamma}(z)=<\frac{m^{2}}{\nu(m)-i z}>_{s}-\left(i z<\frac{\nu(m)}{[\nu(m)-i z]}>_{s}\right)^{-1}\left(<\frac{m}{\nu(m)-i z}>_{s}\right)^{2}
$$

If $P(m)$ and $\nu(m)$ are even functions, then :

$$
<\frac{m}{\nu(m)-i z}>_{s}=0
$$

or, equivalently,

$$
<m \exp (-\nu(m) t)>_{s}=0
$$

and (11) simplifies to :

$$
\tilde{\Gamma}(z)=<\frac{m^{2}}{\nu(m)-i z}>_{s}
$$

The covariance of the KP is then : 


$$
\tilde{\Gamma}(t)=\int_{-\infty}^{+\infty} m^{2} P(m) \exp (-\nu(m)|t|) d m
$$

i.e. the ordinary variance of $P(m)$ conditioned by the probability $\exp (-\nu(m)|t|)$ that no jump occurs between 0 and $t$. Given $P(m)$ and the covariance $\tilde{\Gamma}(t)$, the jumping frequency $\nu(m)$ can be obtained as follows. Let us assume that $\nu(m)$ is a monotonic increasing function of $|m|$ such that $\nu(\infty)=\infty$. Then, taking $\nu$ as a new integration variable, one obtains :

$$
\tilde{\Gamma}(t)=2 \int_{\nu(0)}^{+\infty} m^{2} P(m) \frac{d m}{d \nu} \exp (-\nu|t|) d \nu \quad,
$$

Calculation of $\nu(m)$ requires then the inversion of the Laplace transformation and the solution of a simple differential equation.

It is always possible to construct the KP with an arbitrary probability distribution $P(m)$ and a quite arbitrary covariance $\tilde{\Gamma}$. For the exponential correlations :

$$
\tilde{\Gamma}\left(\left|t-t^{\prime}\right|\right) \equiv<m(t) m\left(t^{\prime}\right)>=\exp \left(-\nu_{0}\left|t-t^{\prime}\right|\right) \quad,
$$

we have :

$$
\nu(m)=\operatorname{const}(m)=\nu_{0}
$$

For the most interesting, algebraic correlations :

$$
\tilde{\Gamma}\left(\left|t-t^{\prime}\right|\right) \equiv<m(t) m\left(t^{\prime}\right)>=\frac{\Gamma(1 / n)}{\left|t-t^{\prime}\right|^{1 / n}} \quad(n>0),
$$

we have [18] :

$$
\nu(m)=\left(2 \int_{0}^{|m|} m^{2} P(m) d m\right)^{n} .
$$

$\Gamma$ in (19) is the gamma function and $P(m)$ is an even function. 


\section{A. One dimensional Kangaroo process}

In the following, we shall assume that both $P(m)$ and $\nu(m)$ are even functions. For $\tilde{\Gamma}(t)=1 / t$, the frequency $\nu(m)$ is :

$$
\nu(m)=2 \int_{0}^{|m|} m^{2} P(m) d m .
$$

The 'free path' length can be defined as $s=1 / \nu$. Since $P(m) d m=P(\nu) d \nu$, therefore :

$$
P(\nu)=P(m)\left(\frac{d \nu}{d m}\right)^{-1}
$$

From (21) we have :

$$
\frac{d \nu}{d m}=2 m^{2} P(m)
$$

and therefore :

$$
P(\nu)=\frac{1}{2 m^{2}} \quad .
$$

The free paths distribution is then :

$$
P(s)=P(\nu) \nu^{2}=\frac{P(\nu)}{s^{2}} .
$$

In order to see whether and how details of the chosen probability density $P(m)$ influence the properties of the KP, let us now consider few simple examples. First, let us take :

$$
P(m)=\frac{2}{m} \quad .
$$

In this case, the jump frequency is : $\nu(m)=2|m|^{2}$ and the frequency distribution : $P(\nu)=$ $\nu^{-1}$. Consequently, the free path distribution becomes :

$$
P(s)=s^{-1}
$$

Next, let us consider :

$$
P(m)=\left\{\begin{array}{lll}
1 / 2 & \text { for } & |m|<1 \\
0 & \text { for } & |m|>1
\end{array}\right.
$$


The jump frequency in this case is : $\nu(m)=\frac{1}{3}|m|^{3}$ and the frequency distribution is : $P(\nu) \sim \nu^{-2 / 3}$. Consequently, the free paths distribution becomes :

$$
P(s) \sim s^{-4 / 3}
$$

Finally, let us consider also the following distribution :

$$
P(m)=\left\{\begin{array}{lll}
2|m|^{-5} & \text { for } & |m|>1 \\
0 & \text { for } & |m|<1
\end{array}\right.
$$

The jump frequency in this case is : $\nu(m)=2\left(1-m^{-2}\right)$ and the frequency distribution is : $P(\nu)=(1-\nu / 2)$. Consequently, the free paths distribution becomes :

$$
P(s) \sim \frac{1}{s^{2}}-\frac{1}{2 s^{3}} \quad .
$$

Therefore, in the limit of long paths : $P(s) \sim s^{-2}$. This is the fastest decaying free paths distribution which can be obtained with the one-dimensional KP. In $N$ dimensions with independent Kangaroo processes in all $N$ directions, the path length distribution becomes : $P_{N}(s)=\left(P_{1}(s)\right)^{N} \sim s^{-\alpha N}$. One should stress however that the norm for such a process : $|\mathbf{m}|=\left(\sum_{i} m_{i}{ }^{2}\right)^{1 / 2}$ is not conserved during the evolution. Moreover, $|\mathrm{m}|$ has not any specific and physically motivated distribution what is a serious drawback of the $(N \times 1)$-dimensional Kangaroo processes.

\section{B. Multidimensional generalization of the Kangaroo process}

In this section we will present the multidimensional generalization of the KP and discuss in details the two-dimensional case. The value of the process is now a vector $\mathbf{m}=\left[m_{1}, m_{2}\right]$ with coordinates $m_{1}, m_{2}$ and a constant norm $:|\mathbf{m}|=1$. Hence, the KP takes random values on a unit circle and the coordinates : $m_{1}=\cos \Theta$ and $m_{2}=\sin \Theta$ are expressed in terms of a single random angle $\Theta$. The covariance of the KP in this case becomes : 


$$
\tilde{\Gamma}(t)=1 / t=2 \int_{\nu(0)}^{\infty} P(\Theta) \frac{d \Theta}{d \nu} \exp (-\nu t) d \nu \quad .
$$

Since $P(\nu) d \nu=P(\Theta) d \Theta$ and $P(\nu)=1$ independently of the form of $P(\Theta)$, therefore :

$$
2 P(\Theta)\left(\frac{d \nu}{d \Theta}\right)^{-1}=1
$$

and :

$$
\nu(\Theta)=2 \int_{0}^{\Theta} P\left(\Theta^{\prime}\right) d \Theta^{\prime}
$$

Obviously, the free path distribution becomes in this case :

$$
P(s)=s^{-2} \quad \text {. }
$$

The function $P(\Theta)$ should satisfy the condition $P(\Theta)=P(\Theta+\pi)$. Generally, it is expedient to sample $\Theta$ only in the interval $[0, \pi / 2]$ and then to extrapolate symmetrically with respect to both axes. The long paths correspond then to $\Theta_{0} \approx 0$ and $\Theta_{1} \approx \pi$. More isotropic distribution $P(\Theta)$ can be obtained by dividing the full angle on sectors of size $\Delta \Theta=\pi / n$, where the integer $n$ may be arbitrarily large. One defines $P(\Theta)$ only in the interval $[0, \pi / n]$ and then chooses the sector with the uniform probability. In this case the long paths will be found at around $\Theta_{k}=\pi k / n(k=0,1, \ldots, 2 n-1)$. If $n=4$ then the free path distribution is similar as in the PLG with the infinite horizon for which long paths are found at $\Theta \approx 0(\approx \pi)$ and $\Theta \approx \pi / 2(\approx 3 / 2 \pi)$. The precise value of $n$ depends on the physical problem considered and in particular on the geometry involved in this problem. For example, in the fission-fusion dynamics with the elongation parameter as a collective variable, one expects that the long path distribution should be strongly asymmetric and hence $n=2$.

The above stochastic process can be easily generalized to still higher dimensions. For example in three dimensions, one has two angles : $\Theta, \phi$, as independent variables. If $\nu=\nu(\Theta)$, independently of the angle $\phi$, then the covariance of the KP becomes :

$$
\tilde{\Gamma}(t)=1 / t=2 \int_{\nu(0)}^{\infty} P(\Theta) P(\phi) \exp [-\nu(\Theta) t] \sin \Theta d \Theta d \phi \quad,
$$


where $P(\Theta) \sin \Theta(d \Theta / d \nu)=1$ and

$$
\nu(\Theta)=\int_{0}^{\Theta} P\left(\Theta^{\prime}\right) \sin \Theta^{\prime} d \Theta^{\prime}
$$

is a uniformly distributed random number. Obviously, as in the above two-dimensional $\mathrm{KP}$, the free path distribution is $P(s)=s^{-2}$. The same holds for an arbitrary number of dimensions providing the frequency $\nu$ of the KP depends only on one angle. With this assumption we impose 'spherical symmetry' into the multidimensional KP which in this way simulates closely the multidimensional extended Sinai billiard (the PLG) problem with spherically symmetric scatterers.

The multidimensional generalized KP can be also easily applied to generate stochastic processes with any algebraic covariance $\tilde{\Gamma}(t) \sim|t|^{-1 / n} \quad(n>0)$. In two dimensions, the frequency $\nu$ of the stochastic process becomes :

$$
\nu=2\left(\int_{0}^{\Theta} P\left(\Theta^{\prime}\right) d \Theta^{\prime}\right)^{n},
$$

and

$$
\frac{d \nu}{d \Theta}=n 2^{n}\left(\int_{0}^{\Theta} P\left(\Theta^{\prime}\right) d \Theta^{\prime}\right)^{n-1} P(\Theta)
$$

Since $P(\nu) d \nu=P(\Theta) d \Theta$, therefore :

$$
P(\nu)=\frac{1}{n 2^{n}}\left(\int_{0}^{\Theta} P\left(\Theta^{\prime}\right) d \Theta^{\prime}\right)^{1-n}=\frac{1}{2 n} \nu^{\frac{1-n}{n}} .
$$

The free paths distribution can now be easily found :

$$
P(s)=\frac{1}{2 n} s^{-\left(1+\frac{1}{n}\right)}
$$

For the exponential covariance (17), the multidimensional norm-conserving generalization of the KP is trivial. Hence, we have succeeded to formulate the Markovian multidimensional process which can approximate multidimensional extended Sinai billiard (the PLG) both in the situation of the closed horizon when the correlations are exponential as well as in the situation of the open horizon when correlations are algebraic. 


\section{LANGEVIN PROBLEM FOR THE MARKOVIAN STOCHASTIC FORCE}

Let us now consider a dissipative system which consists of many particles. Each particle in the system obeys an intrinsic damping which is independent of the fluctuation term. The stochastic force $\mathbf{F}(t)$ acting on a particle in the dissipative system is $\mathbf{F}(t)=\varepsilon \mathbf{m}$, where $\mathbf{m}=\left[m_{1}, m_{2}\right]$ is the value of the KP and $\varepsilon$ is a constant force amplitude [25] .

We shall consider the motion of Brownian particle in the circular attractive potential defined as :

$$
\begin{array}{llll}
V(r)=V_{0}\left(1-\left(\frac{|\mathbf{r}|}{r_{B}}\right)^{2}\right) & \text { for } & & |\mathbf{r}| \leq r_{B} \\
V(r)=0 & \text { for } & |\mathbf{r}|>r_{B},
\end{array}
$$

where $V_{0}$ and $r_{B}$ are the depth and the radius of the potential, respectively. Inside the potential, the motion of particle is given by the Langevin equation (1) with the correlated stochastic force $\mathbf{F}(t)$. Otherwise the particle is free. Initially $(t=0)$, the particle rests at the bottom of the well $(|\mathbf{r}|=0)$. At later times, the stochastic force $\mathbf{F}(t)$ accelerates the particle which may eventually escape from the well. At each jump in the two-dimensional KP, the direction of the vector $m$ changes what corresponds to the update of $\mathbf{F}(t)$. Otherwise the value of the force remains constant. The length of the vector $\mathbf{m}$ is $|\mathbf{m}|=1$ and remains constant.

The quantities of interest are the energy distribution of escaping particles $P(E)$ and the survival-time distribution $N(t)$. The energy distribution of the Langevin particles escaping from the potential well is shown in Fig. 1 both in the case when the stochastic force is generated by the adjoint PLG (the short-dashed line) and in the case when the generalized $\mathrm{KP}$ is applied for this purpose. In the latter case, we consider the two-dimensional generalization of the KP (sect. III.B) for two kinds of the probability distribution functions : $P(\Theta)=P(\Theta+k \pi / n) \quad(k=1, \ldots, n-1)$ with (i) $n=2$ (the long-dashed line) for which the long paths are close to $0, \pi$ and with (ii) $n=4$ (the solid line) for which the long paths are near $0, \pi$ and $\pi / 2,3 / 2 \pi$ directions, like in the PLG. 
In spite of important differences in the definition of the stochastic force generator, the three curves exhibit similar features such as for example the appearance of the peak for 'pre-randomized' particles [3] , which is a characteristic feature of the Langevin approach with long-time correlated noise and corresponds to the 'long free path', i.e. the long time-interval (small $\nu(m)$ ) between the subsequent changes of $\mathbf{m}$ in the stochastic force generator. The Brownian particle escapes as soon as the long free path ends. The second very important qualitative similarity between Markovian (the generalized KP) and nonMarkovian (the PLG) generators corresponds to the Gaussian shape of the energy tail for randomized particles which is a benchmark of the algebraic $(\sim 1 / t)$ velocity and force autocorrelation functions. The details of this Gaussian bump $P(E) \sim \exp \left(-E^{2} / 2 \sigma^{2}\right)$ as quantified by the width parameter $\sigma$ are obviously different for those different generators and equal $\sigma=30.25$ for the non-Markovian generator, and $\sigma=47.6,43.4$ for $n=2$, $n=4$ for the Markovian generators, respectively. We have checked that the width of the Gaussian bump remains almost unchanged when increasing $n$ above $n=4$.

As said above, the existence of the peak for pre-randomized particles is related to the existence of long free paths. Its sharpness is due to the assumed norm conservation in the generalized KP. The qualitative features would remain the same if we would allow for an independent variations of the norm $|\mathrm{m}|$ from a given distribution, say Gaussian.

The escape from the potential requires acceleration by the stochastic force to climb the well. Frequent changes of the applied force reduce the mean acceleration. In the absence of an external potential $V(r)$ or in the case of a small amplitude stochastic force, all trajectories generated either by the PLG for the deterministic random force generator or by the KP for the stochastic generator, both long and short ones, provide a sufficient change of the momentum of the Brownian particle to allow its escape. All those particles which appear sufficiently close to the absorbing barrier at $r=r_{B}$, may escape. The survival probability of the system is thus proportional to the number of particles $N(t)$, still inside the potential well at time $t$. The time-variation of this number depends only on the phase-space density of particles which is just proportional to the number of particles : $\dot{N}(t) \sim N(t)$, i.e. 
$N(t) \sim \exp (-a t)$ where $a$ is a constant.

Situation is quite different for a large amplitude random force. Here the relative number of long free paths increases rapidly in time. The constant random force and the conservative force from the potential act like the gravitation and elastic forces in the problem of the oscillatory motion of a string. The friction force slows down the Brownian particle which finally stops at the point where the constant random force compensates exactly the conservative force [26]. Since the Brownian particle on such trajectories cannot escape, therefore their weight in the ensemble increases in time. The Brownian particle is at standstill or moves on a quasi-periodic orbit as long as the particle stays on a free path. In this case, the balance of forces ensures that the Brownian particle remains inside the absorbing barrier. When the long path finishes, the balance of forces changes and the Brownian particle escapes immediately. Therefore, the particle remains inside the potential well until time $t$ if the long path in the adjoined generator is longer than $t$. Hence, for the Markovian generator (the generalized KP) for which the path length is independent of the length of the previous path, the decay probability for the Brownian particle is for large times proportional to the path length distribution $P(s)$. Therefore, the survival probability until time $t$ is :

$$
P(t) \sim \int_{t}^{\infty} P(s) d s
$$

and the path length distribution is directly related with the survival time distribution inside the potential well.

For the one-dimensional KP with $P(m)$ given by (24), (26) or (28), survival probability distribution $N(t)$ is const $(t), t^{-1 / 3}$ or $t^{-1}$ respectively. Independent KP's in $N$ directions, yield $P(t) \sim t^{1-\alpha N}$ where $\alpha$ is the exponent of the algebraic path distribution in the corresponding one-dimensional KP. It is interesting to notice that the process (24) yields in two dimensions $P(s) \sim s^{-2}$ and hence $P(t) \sim t^{-1}$.

Multidimensional, norm-conserving generalization of the KP, as discussed in sect. III.B, yields $P(s) \sim s^{-2}$, independently of the dimensionality of the problem and independently of the degree of isotropy in the long path distribution. Hence, in this case : $P(t) \sim t^{-1}$, like 
for the non-Markovian generator based on the PLG with the open horizon. Fig. 2 shows the number of surviving Brownian particles inside the spherically symmetric potential both in the case when the stochastic force is generated by the adjoint PLG (the short-dashed line) and in the case when it is generated by the generalized KP in two-dimensions with $n=4$ (the solid line). The line $1 / t$ is shown with the long-dashed line for comparison.

\section{CONCLUSIONS}

The Langevin approach provides a useful framework in which complicated multidimensional Hamiltonian problems can be changed into the low-dimensional dissipative problems, providing one can separate slow, 'collective' degress of freedom from remaining, fast variables. In this case, the collective motion is treated as a Brownian particle embedded in the heat bath introducing fluctuations around the most probable 'macroscopic' collective path. Influence of this 'environment' of fast variables on the slow variables is then taken into account by introducing the noise or the stochastic force. Properties of such a force, in particular its autocorrelation function, must be properly adjusted to fit the 'phenomenological' data (e.g. the CMD data) for a given choice of macroscopic or collective degrees of freedom. Recent CMD studies for the peripheral collisions of ions showed that the local force acting on the 'elementary' particle in the CMD is correlated algebraically : $\tilde{C}(t) \sim t^{-1}$ and is associated with the presence of long free paths. This universal behaviour can be described in the Langevin framework including algebraic correlated stochastic force. In the earlier exploratory studies [3], we have designed the generator of such a stochastic force applying the velocity series of a point particle in the two-dimensional PLG as a generating process of the deterministic, chaotic random process. In case of the open horizon, the velocity autocorrelation function of the particle in the PLG is proportional to $1 / t$ and identifying the time series $\left\{\mathbf{u}\left(t_{0}\right), \mathbf{u}\left(t_{1}\right) \ldots\right\}$ with the time-series $\left\{\mathbf{F}\left(t_{0}\right), \mathbf{F}\left(t_{1}\right) \ldots\right\}$ of the stochastic force $(\mathbf{F}(t) \sim \mathbf{u}(t))$, one obtains the non-Markovian generator of the stochastic force acting on the Brownian particle. This generator has desired correlation properties but its practi- 
cal implementation may be cumbersome. Hence, in this work we have studied Markovian generators of the stochastic force which are based on the KP. In particular, we have proposed a special, multidimensional generalization of the KP, conserving the norm and having the covariance $\tilde{\Gamma}(t) \sim t^{-1}$ as the PLG process for the open horizon case. We have found also that the path length distribution, which is $P(s) \sim s^{-3}$ for the non-Markovian PLG case independently of the dimensionality, equals $P(s) \sim s^{-2}$ in the generalized $\mathrm{KP}$, also independently of the dimensionality of the problem. This difference is however not essential for the properties of the Brownian particles. In particular, both the survival probability for the Brownian particle to remain inside of the potential as well as the asymptotic energy distribution of particles are qualitatively the same and can be made almost identical by an appropriate change of the geometry of the PLG, i.e. by changing the radii $R$ of the circular scatterers. These result remain unchanged if one allows variations of $|\mathbf{m}|$ (or $|\mathbf{u}|$ in the case of the PLG) of the stochastic process. Hence, we have constructed a reliable and simple generator of the long-time correlated stochastic process which in the particular case of the $1 / t$-covariance, is equivalent to the deterministic $1 / t$-correlated process in the PLG. The advantage of the Markovian generator lies in its flexibility to describe physical situations with a different degree of isotropy in the distribution of the long free path. One should also stress that both for the Markovian and non-Markovian generators, the long free paths are responsible for the appearance of the algebraic covariance of the process. An interesting special case of the Markovian generator corresponds to $P(m)$ of $(28)$ in one dimension. In this case, the KP has long free paths and the $1 / t$ correlations, like for the PLG (the extended Sinai billiard). In application to the Langevin problem, it yields the survival probability distribution : $N(t) \sim 1 / t$. However, contrary to the PLG, this one-dimensional Markovian analogue of the billiard has a variable size $m$ of the stochastic process. 


\section{Acknowledgements}

The authors wish to thank Y. Abe, S. Ayik for their interest in the present work and useful comments. The work was partly supported by KBN Grant No. 2 P03 B 14010 and the Grant No. 6044 of the French - Polish Cooperation. 


\section{REFERENCES}

[1] S. Drożdż, J. Okołowicz and M. Płoszajczak, Phys. Lett. 109B (1982), 145;

E. Caurier, B. Grammaticos and T. Sami, Phys. Lett. 109B (1982), 150;

W. Bauhoff, E. Caurier, B. Grammaticos and M. Płoszajczak, Phys. Rev. C32 (1985), 1915;

H. Feldmeier, Nucl. Phys. A515 (1990), 147;

A. Ono, H. Horiuchi, T. Maruyama and A. Ohnishi, Phys. Rev. Lett. 68 (1992), 2898; Prog. Theor. Phys. 87 (1992), 1367.

[2] T. Srokowski and M. Płoszajczak, Phys. Rev. Lett. 75 (1995), 209.

[3] M. Płoszajczak and T. Srokowski, GANIL Preprint P 95 25, Ann. Phys. (N.Y.) (in print).

[4] T. Srokowski, J. Okołowicz, S. Drożdż and A. Budzanowski, Phys. Rev. Lett. 71 (1993), 2867;

S. Drożdż, J. Okołowicz, T. Srokowski and A. Budzanowski, Phys. Rev. C51 (1995), 2630.

[5] A. Zacherl, T. Geisel, J. Nierwetberg and G. Radons, Phys. Lett. 114A (1986), 317.

[6] D.R. Hofstadter, Phys. Rev. B14, 2239 (1976).

[7] T. Geisel, J. Wagenhuber, P. Niebauer and G. Obermair, Phys. Rev. Lett. 64, 1581 (1990).

[8] S. Drożdż, J. Okołowicz, M. Płoszajczak and T. Srokowski, GANIL preprint P 9609.

[9] H.A. Kramers, Physica VII 4 (1940), 284.

[10] H. Mori, Prog. Theor. Phys. 33 (1965), 423;

R. Kubo, Rep. Prog. Phys. 29 (1966), 255.

[11] P. Hänggi and P. Jung, Adv. Chem. Phys. 89 (1995), 229.

[12] R. F. Grote and J. T. Hynes, J. Chem. Phys. 73 (1980), 2715.

[13] P. Hänggi, J. Stat. Phys. 30 (1983), 401.

[14] A. Igarashi and T. Munakata, J. Phys. Soc. Jpn. 57 (1988), 2439.

[15] A. Brissaud and U. Frisch, J. Quant. Spectrosc. Radiat. Transfer 11 (1971), 1767.

[16] R. Kubo, J. Math. Phys. 4 (1962), 174.

[17] P. W. Anderson, J. Phys. Soc. J. 9 (1954), 316;

R. Kubo, J. Phys. Soc. J. 9 (1954), 935. 
[18] A. Brissaud and U. Frisch, J. Math. Phys. 15 (1974), 524.

[19] Ya. G. Sinai, Russ. Math. Surv. 25 (1970), 137.

[20] M. V. Berry, Eur. J. Phys. 2 (1981), 91.

[21] J.P. Bouchaud and P.L. Le Doussal, J. Stat. Phys. 41 (1985), 225.

[22] B. Friedman and R. F. Martin, Phys. Lett. A105 (1984), 2.

[23] A. Brissand and U. Frisch, J. Quant. Spectrosc. Radiat. Transfer 11 (1971), 1767.

[24] A.T. Bharucha-Reid, Elements of the Theory of Markov Processes and Their Applications (McGraw \& Hill, New York, 1960).

[25] In studies using the two-dimensional PLG as a generator of a 'deterministic' random force acting on a Brownian particle, $\mathbf{F}(t)=\varepsilon \mathbf{u}$, where $\mathbf{u}(|\mathbf{u}|=1)$ is the velocity of particle in the PLG and $\varepsilon$ is the force amplitude.

[26] In the absence of friction, the Brownian particle evolves on a quasi-periodic orbit. 


\section{Figure captions}

\section{Fig. 1}

The asymptotic energy distribution of the particles escaping from the circular attractive potential of depth $V_{0}=-40$ and radius $r_{B}=50$ for the stochastic force with the covariance proportional to $1 / t$. The constant of intrinsic friction is $\gamma=0.02$. The short-dashed line shows the results for the stochastic force generated by the deterministic, chaotic rule of the PLG. The long-dashed and solid lines exhibit results for the generalized KP in two dimensions with $n=2$ and $n=4$ respectively (see sect. III.B). In this case, the probability distribution functions are periodic functions : $P(\Theta)=P(\Theta+k \pi / n)$ with $(k=1, \ldots, n-1)$ on a circle of radius $|\mathrm{m}|=1$ and the long free paths are concentrated at around $\Theta_{k}=k \pi / n$.

\section{Fig. 2}

The variation of the particle number inside the circular attractive potential of depth $V_{0}=-50$ and radius $r_{B}=50$. The absorbing barrier is at $r=r_{B}$. The intrinsic friction constant is $\gamma=0.02$. The short-dashed line has been obtained for the deterministic chaotic force generated by the PLG with the open horizon. The solid line corresponds to the calculations performed with the two-dimensional generalized KP with $n=4$ (see sect. III.B). In both case, the stochastic force has the covariance proportional to $1 / t$. For more details see the description in the text. 


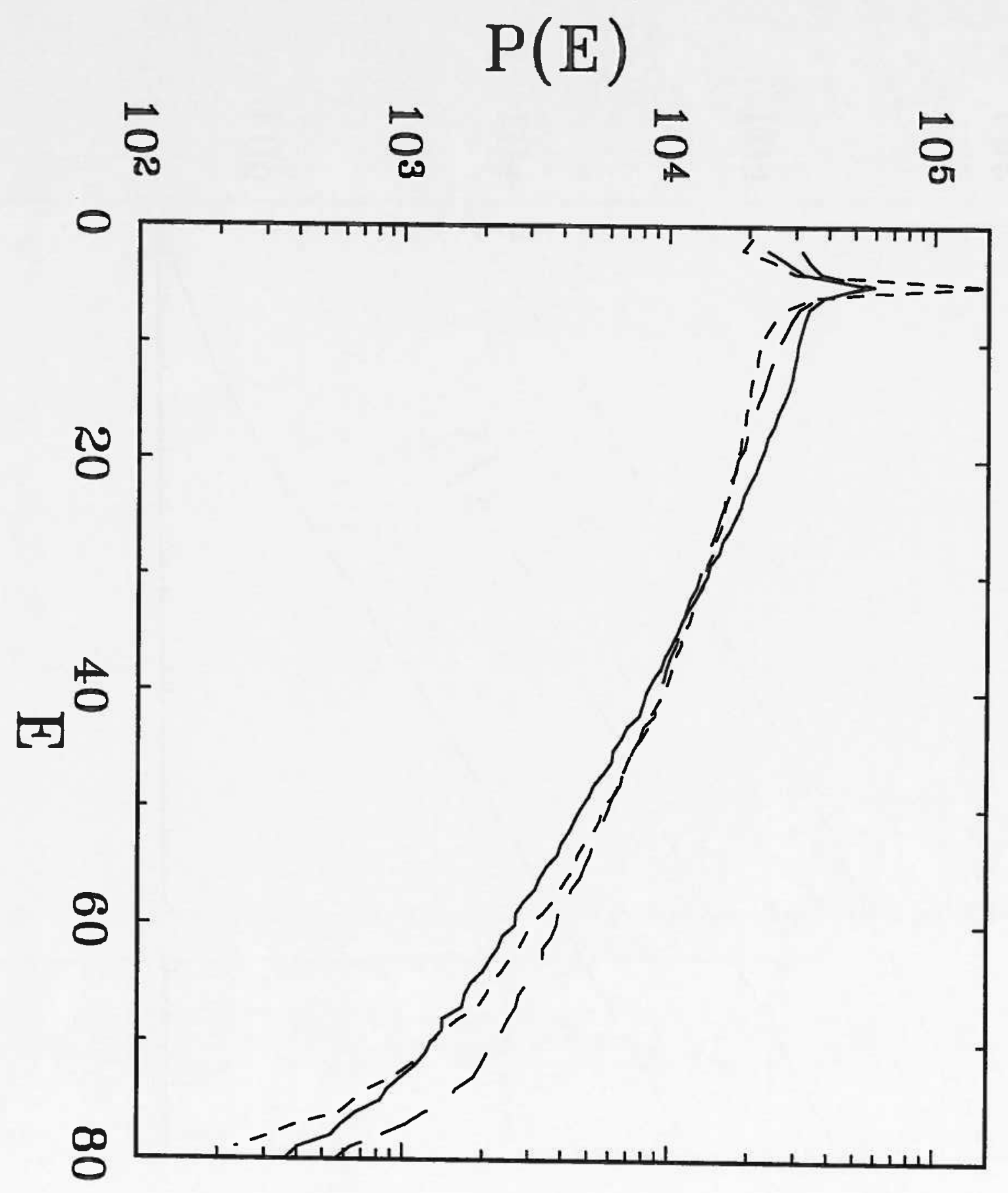

Fig. 1 


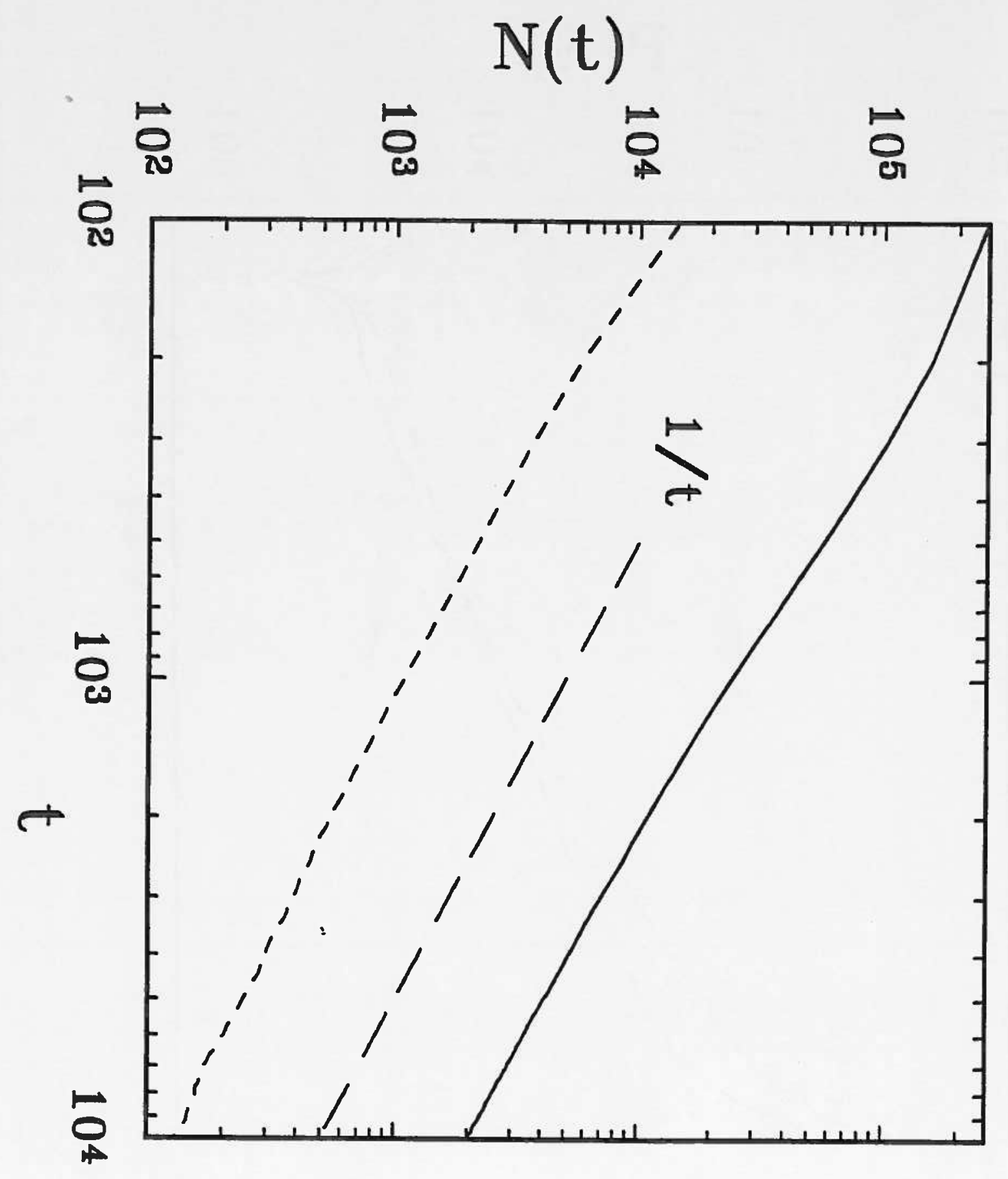

Fig. 2 